\title{
Publisher Correction: Cortical encoding of speech enhances task-relevant acoustic
} information

Sanne Rutten (D), Roberta Santoro, Alexis Hervais-Adelman (D), Elia Formisano (D) and Narly Golestani (D)

Correction to: Nature Human Behaviour https://doi.org/10.1038/s41562-019-0648-9, published online 8 July 2019.

In the version of this article initially published, the $x$ axis in the left panel of Fig. 1b was mislabeled: the rightmost tick mark should have been labeled 4.0. The error has been corrected in the HTML and PDF versions of the article.

Original

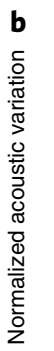

Spectral profile

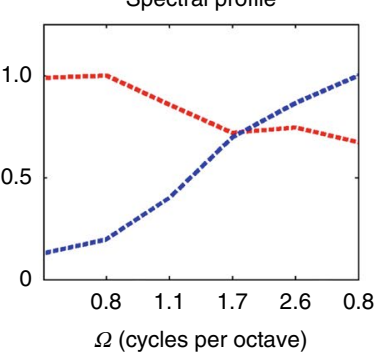

Corrected

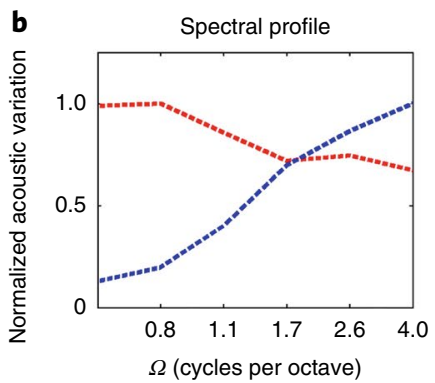

Fig. 1 | Orginal and Corrected. 